\title{
Radio Detection of Cosmic Rays with LOPES (LOPES Collaboration)
}

\author{
C. Grupen, ${ }^{1}$ W.D. Apel, ${ }^{2}$ T. Asch, ${ }^{3}$ A.F.Badea,${ }^{2,4}$ L. Bähren, ${ }^{5}$ K. Bekk, ${ }^{2}$ A. Bercuci, ${ }^{6}$ M. Bertaina, ${ }^{7}$ P.L. Biermann, ${ }^{8}$ \\ J. Blümer, ${ }^{2,9}$ H. Bozdog, ${ }^{2}$ I.M. Brancus, ${ }^{6}$ S. Buitink, ${ }^{10}$ M. Brüggemann, ${ }^{1}$ P. Buchholz, ${ }^{1}$ H. Butcher, ${ }^{5}$ A. Chiavassa, ${ }^{7}$ \\ F. Cossavella, ${ }^{9}$ K. Daumiller, ${ }^{2}$ F. Di Pierro, ${ }^{7}$ P. Doll, ${ }^{2}$ R. Engel, ${ }^{2}$ H. Falcke,${ }^{5,8,10}$ H. Gemmeke, ${ }^{3}$ P.L. Ghia, ${ }^{11}$ R. Glasstetter, ${ }^{12}$ \\ A. Haungs, ${ }^{2}$ D. Heck, ${ }^{2}$ J.R. Hörandel, ${ }^{9}$ A. Horneffer, ${ }^{10}$ T. Huege,${ }^{2}$ K.H. Kampert, ${ }^{12}$ Y. Kolotaev, ${ }^{1}$ O. Krömer, ${ }^{3}$ J. Kuijpers, ${ }^{10}$ \\ S. Lafebre, ${ }^{10}$ H.J. Mathes, ${ }^{2}$ H.J. Mayer, ${ }^{2}$ C. Meurer, ${ }^{2}$ J. Milke, ${ }^{2}$ B. Mitrica, ${ }^{6}$ C. Morello, ${ }^{11}$ G. Navarra, ${ }^{7}$ S. Nehls, ${ }^{2}$ \\ A. Nigl, ${ }^{10}$ R. Obenland, ${ }^{2}$ J. Oehlschläger, ${ }^{2}$ S. Ostapchenko, ${ }^{2,13}$ S. Over, ${ }^{1}$ M. Petcu, ${ }^{6}$ J. Petrovic, ${ }^{10}$ T. Pierog, ${ }^{2}$ S. Plewnia, ${ }^{2}$ \\ H. Rebel, ${ }^{2}$ A. Risse, ${ }^{14}$ M. Roth,${ }^{2}$ H. Schieler, ${ }^{2}$ O. Sima,${ }^{6}$ K. Singh, ${ }^{10}$ M. Stümpert, ${ }^{9}$ G. Toma, ${ }^{6}$ G.C. Trinchero, ${ }^{11}$ \\ H. Ulrich, ${ }^{2}$ J. van Buren, ${ }^{2}$ W. Walkowiak, ${ }^{1}$ A. Weindl, ${ }^{2}$ J. Wochele, ${ }^{2}$ J. Zabierowski, ${ }^{14}$ J.A. Zensus, ${ }^{8}$ and D. Zimmermann ${ }^{1}$ \\ ${ }^{1}$ Siegen University, Department of Physics, Emmy-Noether-Campus, Walter-Flex-Strasse 3, D-57068 Siegen, Germany \\ ${ }^{2}$ Institut für Kernphysik, Forschungszentrum Karlsruhe, 76021 Karlsruhe, Germany \\ ${ }^{3}$ Institut für Prozessdatenverarbeitung und Elektronik, Forschungszentrum Karlsruhe, 76021 Karlsruhe, Germany \\ ${ }^{4}$ on leave of absence from National Institute of Physics and Nuclear Engineering, Bucharest, Romania \\ ${ }^{5}$ ASTRON, 7990 AA Dwingeloo, The Netherlands \\ ${ }^{6}$ National Institute of Physics and Nuclear Engineering, 7690 Bucharest, Romania \\ ${ }^{7}$ Dipartimento di Fisica Generale dell' Universita, 10125 Torino, Italy \\ ${ }^{8}$ Max-Planck-Institut für Radioastronomie, 53121 Bonn, Germany \\ ${ }^{9}$ Institut für Experimentelle Kernphysik, Universität Karlsruhe, 76021 Karlsruhe, Germany \\ ${ }^{10}$ Department of Astrophysics, Radboud University, 6525 ED Nijmegen, The Netherlands \\ ${ }^{11}$ Istituto di Fisica dello Spazio Interplanetario, INAF, 10133 Torino, Italy \\ ${ }^{12}$ Fachbereich C - Physik, Universität Wuppertal, 42097 Wuppertal, Germany \\ ${ }^{13}$ on leave of absence from Moscow State University, 119899 Moscow, Russia \\ ${ }^{14}$ Soltan Institute for Nuclear Studies, 90950 Lodz, Poland \\ Received on 10 March, 2006
}

\begin{abstract}
Data taken with a radio antenna array in combination with the ground-level air shower experiment KASCADE-Grande at the Forschungszentrum Karlsruhe open up the possibility to measure large extensive air showers with this new technique. The pulse height of the observed radio signals scales with the primary energy of the particles initiating the air shower. The dependence of the radio signal on the angle of the shower axis with respect to the Earth's geomagnetic field and the coherence of the radiation suggest that the radio signal generation is due to the geosynchrotron mechanism.
\end{abstract}

Keywords: Cosmic rays; Extensive air shower; Radio emission

\section{INTRODUCTION}

The composition, origin, acceleration mechanism, and the propagation of the most energetic cosmic rays is still an unsolved puzzle. The solution to these problems is complicated by the fact that ultra high energy cosmic rays are extremely rare. Only one particle per square kilometer and century arrives at Earth with energies in excess of $10^{20} \mathrm{eV}$. Apart from the intensity also 'classical particles' like protons and photons have difficulties in arriving from large distances. Photons are significantly attenuated by gamma-gamma interactions with blackbody, infrared, or optical photons, like $\gamma+\gamma \rightarrow e^{+}+e^{-}$, leading to typical attenuation lengths of $\lambda \approx 10 \mathrm{kpc}$. Also energetic protons $\left(E \geq 6 \cdot 10^{19} \mathrm{eV}\right)$ undergo photonuclear interactions with the numerous blackbody photons, like $\gamma+p \rightarrow$ $\Delta^{+} \rightarrow n+\pi^{+}$or $\gamma+p \rightarrow \Delta^{+} \rightarrow p+\pi^{0}$, thereby losing a large part of their energy. Typical attenuation lengths for these processes are $\lambda \approx 10 \mathrm{Mpc}$. The consequence is that in the light of photons and protons (even more so for heavier nuclei) only the local universe is visible. Neutrinos would in principle provide a way out of this dilemma. However, their detection involves considerable experimental difficulties [1].

Because of intensity reasons it is impossible to detect high energy cosmic rays ( $E \geq 1 \mathrm{PeV}$ ) outside the Earth's atmosphere. Detectors with sufficient collection power cannot be flown on satellites. Therefore an effective detection of air showers, initiated by energetic primaries, at mountain altitudes or ground level becomes mandatory.

Classical sampling techniques, like the KASCADE [2] or AGASA [3] arrays cover only a small fraction of the area mostly in the tail of the cascade developing in the atmosphere. Air fluorescence telescopes like HiRes [4] or those of the Auger experiment [5] have the advantage of recording the complete longitudinal development of the air shower in the atmosphere, albeit at the expense of a low duty cycle $(\approx 10 \%)$, because the detection of the feeble scintillation light requires clear, moonless nights. If these air showers could also be detected in the radio band, the advantage of getting the full 
shower size by integrating over the longitudinal development in the atmosphere could be combined with a near $100 \%$ duty time. In addition, the radio detection can be accomplished at moderate costs in detectors compared to sampling or fluorescence techniques, but maybe not so low at costs in the required network.

\section{GENERATION AND DETECTION OF RADIO SIGNALS}

The majority of air shower particles are electrons, positrons and photons. Initially one third of the energy of the primary particle is transferred to neutral pions which feed the electromagnetic cascade via their two-photon decay. Further electromagnetic energy results from $\pi^{0}$ production in interactions of secondary charged particles $\left(\pi^{ \pm}, K^{ \pm}, \ldots\right)$, so that eventually a large fraction of the primary energy is observed in electrons and positrons. These particles travel with approximately the speed of light through the atmosphere. The thickness of the shower disk moving through the atmosphere is just a few meters (corresponding to $\approx 10 \mathrm{~ns}$ ), so that coherent phenomena are expected for radio frequencies up to a few $100 \mathrm{MHz}$. Since the velocity of the electrons and positrons exceeds the velocity of light in air ( $\mathrm{v}_{\text {electron }} \geq \mathrm{c} / n$, where $n$ is the index of refraction of air), one would expect a coherent production of Cherenkov radiation due to the Askaryan effect [6]. Askaryan argued that in electromagnetic showers an excess of electrons over positrons must develop, because on the one hand positron annihilation removes part of the positive charge while on the other hand Compton and $\delta$ electrons add new negative charge to the shower. For the generation of Cherenkov radiation the effect of negative and positive charges - due to their charge imbalance - would no longer cancel, giving rise to a net Cherenkov signal. This phenomenon is expected to become dominant in dense media, like water or ice. On the other hand the electrons and positrons are deflected in the Earth's magnetic field providing a source of geosynchrotron radiation, which is believed to be the dominant mechanism of radio generation in the atmosphere. In air this process produces a stronger emission than the Askaryan effect, since it involves all of the charged particles, rather than just the excess charge as in the coherent Cherenkov emission [7]. Other generation mechanisms or interpretations like dipole radiation, due to charge separation in the Earth's magnetic field [8], or the effect of molecular-field bremsstrahlung [7] are believed to be of minor importance.

The advantage of radio detection over air shower sampling or air fluorescence detection can be characterised as follows:

- simple, robust, and inexpensive detectors;

- full day operation possible (maybe except thunderstorms);

- integration over the whole air shower development, providing information on the total shower energy;

- wide field of view;

- low attenuation of the radio signal.
The determination of the chemical composition of primary cosmic rays using radio detection is more difficult compared to fluorecence techniques, because the radio signal integrates over the whole shower development, while the fluorescence signal allows also to determine the position of the maximum of the shower development $X_{\max }$, which is sensitive to the nature of the particle that initiates the shower.

Potential problems of the radio technique also consist in radio frequency interference, which, however, can be overcome by digital filtering techniques and beam forming [9-11]. Due to the omnipresent radio frequency noise radio detection is only feasible at high energies $\left(\geq 10^{16} \mathrm{eV}\right)$ where the signalto-noise ratio becomes favourable.

\section{RADIO DETECTION AND THE LOPES ARRAY}

The phenomenon of radio emission from extensive air showers was experimentally discovered in 1965 by Jelley et al. [12]. Early measurements in the sixties were compiled by Allan [13]. Theoretical descriptions of the phenomenon were provided by Kahn and Lerche [8] and Colgate [14] soon after. Many activities developed in the late sixties and early seventies, but due to problems with radio interference, poor time resolution, low statistics, and limited angular acceptance this field of research soon quieted down and became dormant. Only with the availability of modern electronics and the possibility of digital signal filtering and beam forming [9-11, 15] the radio detection of extensive air showers became rejuvenated as a realistic alternative to classical air shower detection. In addition, more comprehensive theoretical descriptions and Monte Carlo codes for the understanding of the phenomenon pushed the field [7, 16-19].

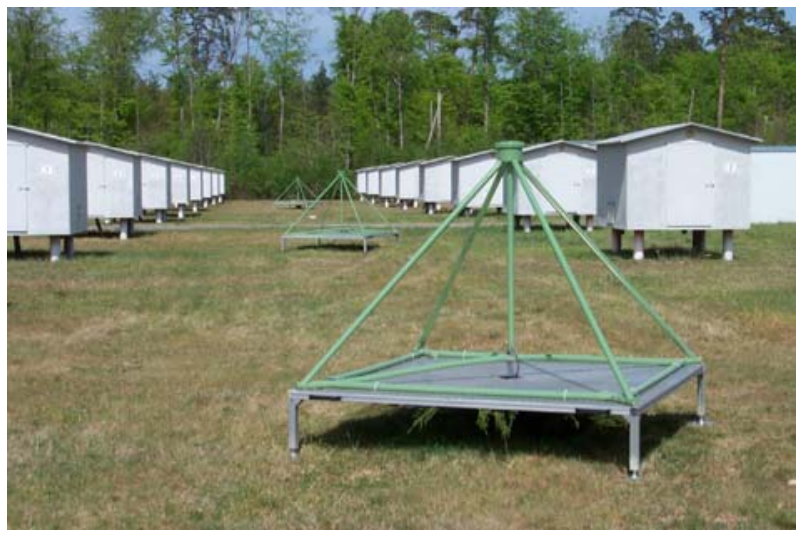

FIG. 1: Inverted V dipole antenna for the LOPES experiment integrated into the KASCADE array. Only two 'legs' of the dipole are equipped with antennas, such that only one polarisation in east-west direction can be measured. In the background one can see some scintillator sampling detectors in wooden housings on the campus of the Forschungszentrum Kalrsruhe.

The LOPES experiment (LOFAR prototype station; LOFAR - Low Frequency Array [20]) is a prototype detector system of a much larger array (LOFAR) to be built in the 
Netherlands predominantly meant for radio astronomy. Initially LOPES comprised 10 simple inverted V dipole antennas (figure 1), called LOPES 10. It was later extended to 30 antennas (LOPES 30). These antennas are installed on the site of the original and extended Karlsruhe air shower array (KASCADE [2] (figure 2) and KASCADE-Grande [21]) at the Forschungszentrum Karlsruhe. KASCADE and KASCADEGrande represent multi-detector setups which allow high performance estimations of charged particles (electrons, photons, muons, and hadrons) in extensive air showers in the primary energy range from $10^{14}$ to $10^{18} \mathrm{eV}$.

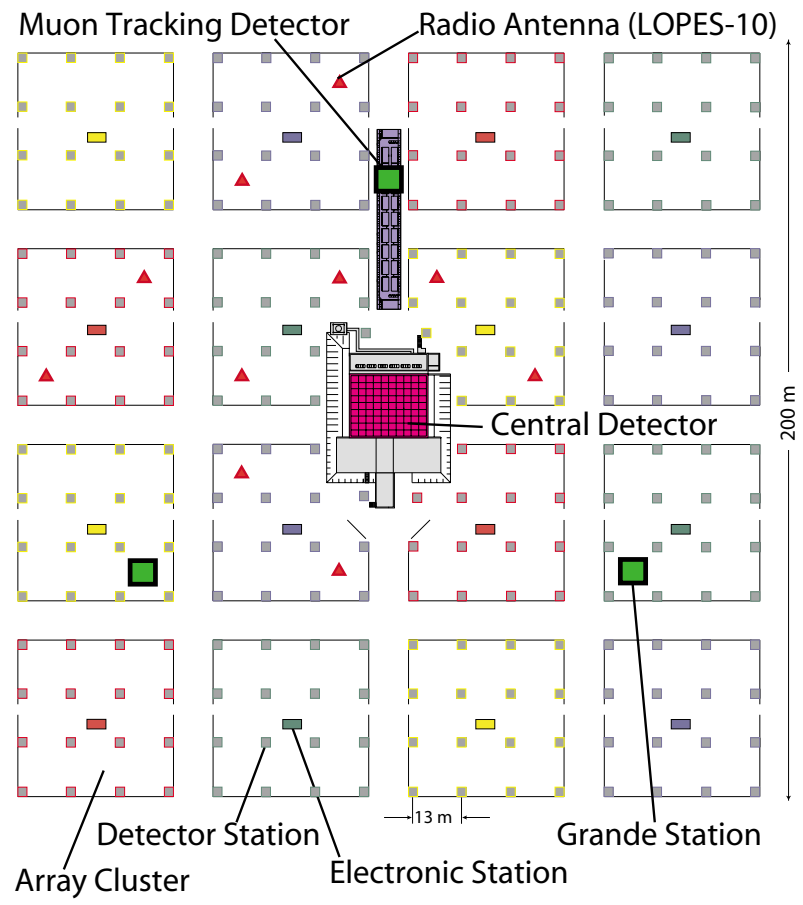

FIG. 2: The main detector components of the KASCADE experiment consisting of 16 clusters of scintillation counters of the field array (in total 252 detector stations), a special muon tracking detector, and a central detector incorporating a large hadron calorimeter.

The 10, respectively 30 LOPES dipole antennas are placed essentially inside the original KASCADE array $(200 \times$ $200 \mathrm{~m}^{2}$ ) with some outside in the KASCADE-Grande detector system $\left(800 \times 800 \mathrm{~m}^{2}\right.$, Fig. 3). The electron and muon components of the air showers are measured in small stations containing several scintillation counters each (Fig. 4).

The antennas of the LOPES detector system operate in the frequency range $40-80 \mathrm{MHz}$. They are aligned in east-west direction and are therefore only sensitive to the east-west linear polarisation component of the radio emission. The readout of the LOPES antennas is triggered by the KASCADE array. The readout window for the antennas is $0.8 \mathrm{~ms}$ wide, and it is centered on the KASCADE trigger. The KASCADE array provides the information on the energy of the primary particle initiating the air shower, the direction of the shower axis, and the distance of the shower axis from the antennas. These data are derived from the lateral distribution of electrons, photons, and muons as observed in the various detectors of the

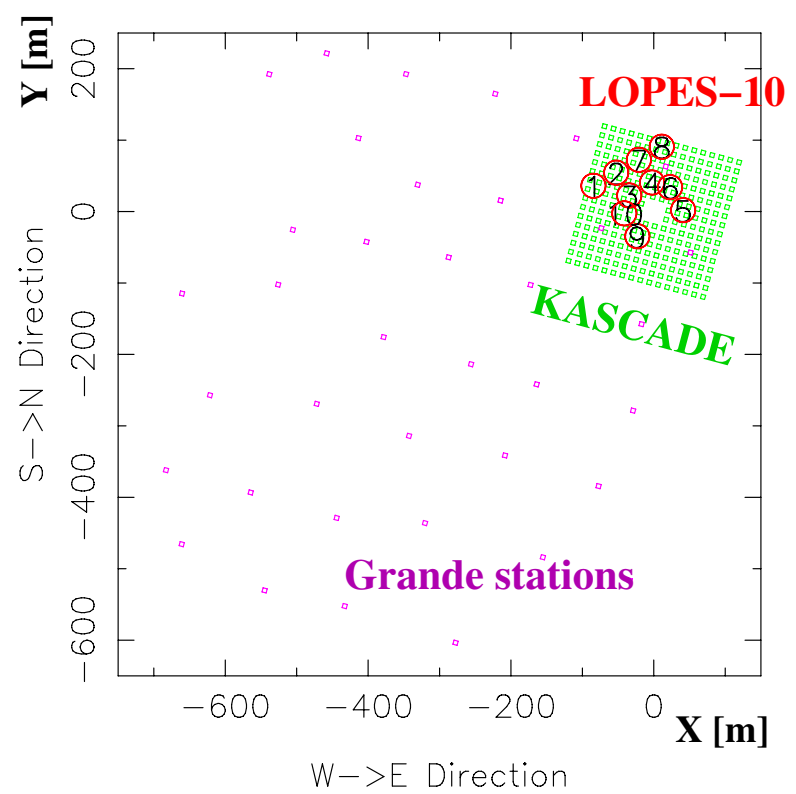

FIG. 3: Sketch of the KASCADE-Grande experiment. KASCADEGrande is an extension of the smaller KASCADE array. It has 37 additional detector stations. The position of the first 10 LOPES antennas is indicated.

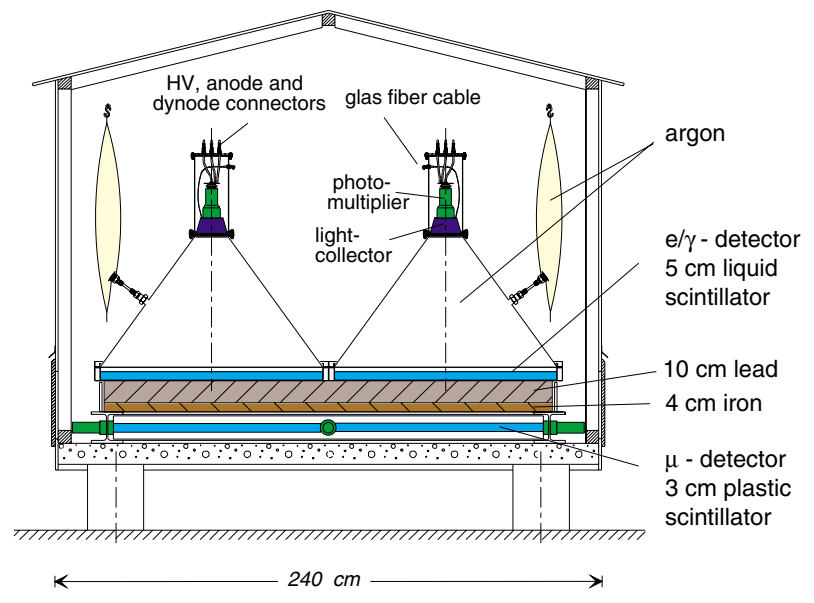

FIG. 4: Cross section of one electron and muon detection unit consisting of scintillation counters in the stations of the KASCADE array.

KASCADE and KASCADE-Grande arrays. The shower size, or the energy of the primary particle, initiating the shower, is usually obtained from the electron component in the air shower, or by a combination of the electron and muon size.

The antennas for the initial LOPES 10 array were not calibrated; however, for LOPES 30 a calibration has been performed with a commercial radio source operating at frequencies between $40 \mathrm{MHz}$ and $80 \mathrm{MHz}$. The radio source was positioned $10 \mathrm{~m}$ above the antennas. The frequency was varied in steps of $1 \mathrm{MHz}, 5 \mathrm{MHz}$, and $10 \mathrm{MHz}$. The signal consisted of a sine wave with a field strength of $E=80 \mu \mathrm{V} /(\mathrm{m} \cdot \mathrm{MHz})$.

According to Allan [13] an estimate of the expected pulse 
amplitude of the radio signal $\varepsilon_{v}$ per unit bandwidth induced by the air shower can be described by the following formula,

$$
\varepsilon_{\mathrm{v}}=20 \cdot \frac{E_{0}}{10^{17} \mathrm{eV}} \cdot \sin \alpha \cdot \cos \theta \cdot \exp \left(-\frac{R}{R_{0}(v, \theta)}\right)\left[\frac{\mu \mathrm{V}}{\mathrm{m} \cdot \mathrm{MHz}}\right],
$$

where

$E_{0}$ - primary energy of the shower in $\mathrm{eV}$,

$\alpha-$ angle between the shower axis and the geomagnetic field,

$\theta-$ zenith angle of the shower,

$R$-distance to the shower axis, and

$R_{0}(v, \theta)=110 \mathrm{~m}$ at $55 \mathrm{MHz}$ is a scaling radius.

This formula, obtained from measurements of small bandwidths, should be considered as a guideline for radio detection. In particular, the numerical scaling factor of 20 has to be considered with caution.

LOPES data have been analysed in many different ways. One of the main aims of the pioneering LOPES experiment was to establish the feasability of radio detection of air showers, and to clarify the production mechanism of radio emission. In special analyses the behaviour of distant events [10], the question of the zenith-angle dependence of radio production, and the possibility for neutrino detection based on radio emission [22] was investigated.

In general, LOPES data are selected by the following cuts:

- trigger from the KASCADE-Grande arrays;

- a successful reconstruction of the air shower with the core position inside the KASCADE-Grande arrays;

- zenith angle smaller than $50^{\circ}$;

- $E_{0} \geq 4 \cdot 10^{16} \mathrm{eV}$;

- distance cut depending on the energy (e.g. $R \leq 200 \mathrm{~m}$ at $10^{17} \mathrm{eV}, R \leq 400 \mathrm{~m}$ at $\left.4 \cdot 10^{17} \mathrm{eV}\right)$;

- approximately uniform pulse height in all antennas.

The analysis in this paper is based on LOPES 10 data. The LOPES 10 antennas are essentially placed inside the original, smaller KASCADE array (see figure 3). Therefore, the analysis relies predominantly on properties given by the original KASCADE set-up. The offline analysis of the LOPES 10 data required a trigger from the traditional, smaller sampling array, and the following cuts were applied [9]:

- the KASCADE event readout was 'good', in the sense that the array processor was working properly;

- the distance of the shower core to the KASCADE array centre was less than $91 \mathrm{~m}$, corresponding to the effective radius of the KASCADE array;

- the electron number was greater than $5 \cdot 10^{6}$, or the truncated muon number (i.e. the number of muons in the distance range of $40-200 \mathrm{~m}$ from the shower axis) was greater than $2 \cdot 10^{5}$.
The last criterion ensures that mainly large events are selected, where a significant radio signal is expected.

The analysis of the data is done offline and proceeds along the following lines:

- correction for instrumental delays;

- gain correction for all electronic components;

- filtering of narrow band radio frequency interference;

- selection of antennas with significant signals;

- digital beam forming in the direction of the shower [911];

- quantification of the peak parameters.

The digital beam forming presents an essential step in the analysis. The signals from the antennas are shifted and crosscorrelated in such a way that the coherent pulse from the air shower is optimised using the information of the zenith angle and azimuthal angle from the KASCADE arrays [9]. Since the bulk of the radio signal originates mainly from the large number of electrons in the shower maximum - i.e. from an extended point source - it is also expected that the radio wavefront, instead of being a plane wave, should have some curvature. During the reconstruction procedure this radius of curvature is taken into account by iterating it as a free parameter until the radio beam is maximal $[10,11]$. This curvature parameter relates essentially to the 'electromagnetic' shower front.

Typical radio events have amplitudes $\varepsilon_{v}$ in the frequency range of $40-80 \mathrm{MHz}$ of some $\mu \mathrm{V} /(\mathrm{m} \cdot \mathrm{MHz})$ corresponding to electrical fields of $E \approx 150 \mu \mathrm{V} / \mathrm{m}$. It has to be noted that at coherence the radio power $P$ is proportional to the square of the electric field.

\section{RESULTS}

The big advantage of radio detection of air showers in a phased array is that radio antennas in principle look in all directions in the sky at the same time. For a known angle of incidence of the air shower - in this case provided by the KASCADE arrays - the signals can be phased in that direction by remotely adjustable delays. Offline many different directions can be tested by the method of phased digital beam forming $[10,11]$. This technique also works without using the information of the shower direction.

The electric field as measured at each antenna corrected for the arrival direction still shows a lot of incoherent noise (see figure 5, [23]).

The noise is due to incoherent radio signals from the KASCADE readout sampling detectors (scintillation counters with photomultiplier readout). The advantage of the crosscorrelated beam forming is that a radio peak from a real coherent pulse always has a positive sign, while peaks from incoherent noise can also have negative sign, so that incoherent pulses present in the signal can cancel. The sum of the delaycorrected electric fields from all antennas squared for the same 
[1] Event1073867291-10101

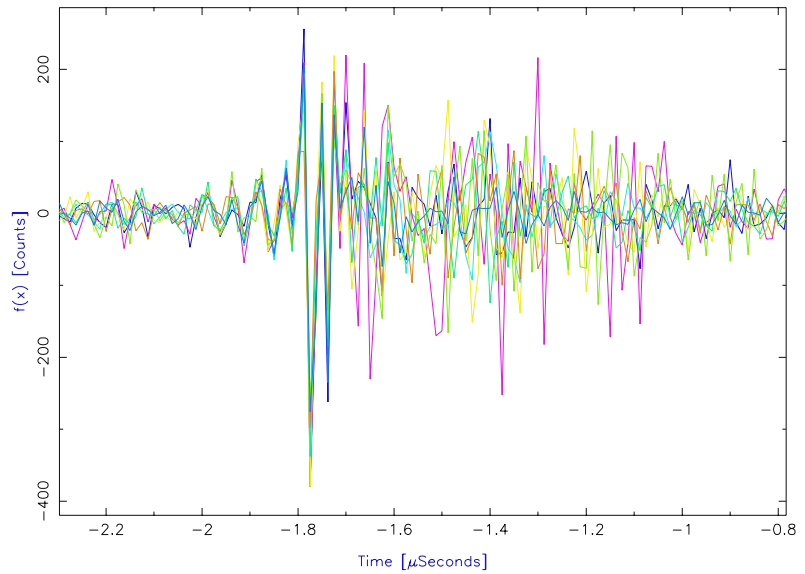

FIG. 5: Superposition of the electric fields of all antennas for an individual shower event. The various pulses are delay-corrected for the arrival direction of the air shower [23].

event as in figure 5 is shown in figure 6 [23]. The time offset of $\approx 1.8 \mu$ s of the radio signal with respect to the KASCADE trigger is due to hard-wired delays.

Figure 7 shows an example of a false colour radio map of an air shower event. By analyzing the arrival times of the radio flashes the direction of incidence of the shower can be independently determined. The result of such a shower reconstruction is seen as central blob in the figure. Other weak signals surrounding the central brightness maximum result from interferometer sidelobes by the sparse radio array and from background noise in the radio signals [15].

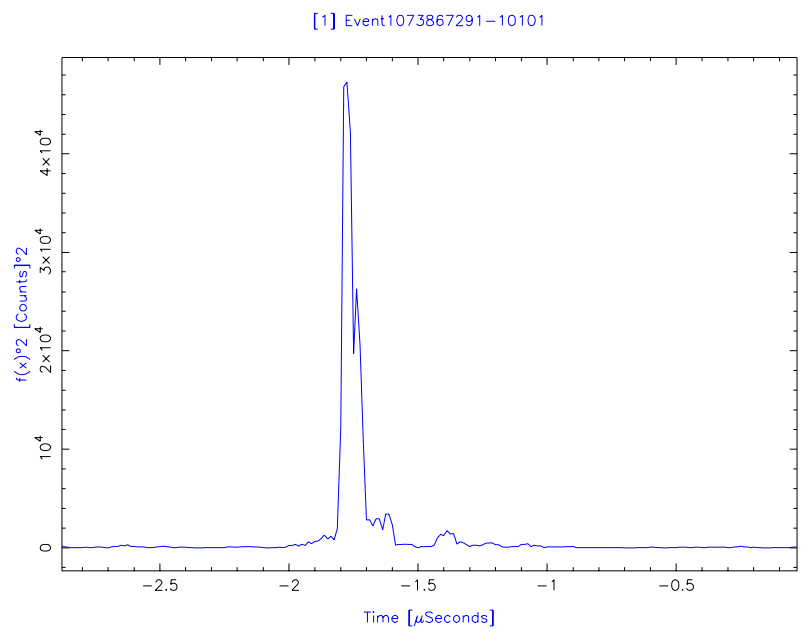

FIG. 6: Sum of delay-corrected electric fields from all antennas squared for an individual event [23].

The pulse height of the radio signal is proportional to the integrated track length of all electrons and positrons in the air shower. It should depend simultaneously on a number of other parameters, namely

- number of muons;

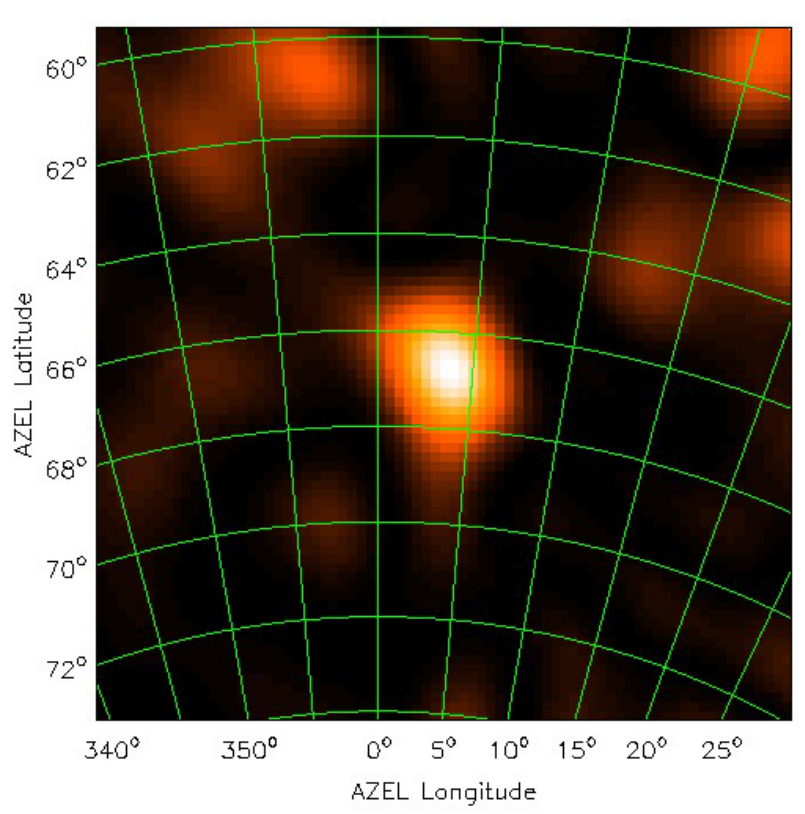

FIG. 7: False colour radio map of an air shower event. The reconstructed image of the shower is seen as bright blob at the centre of the figure. Other weak signals surrounding the central brightness maximum result from interferometer sidelobes by the sparse radio array and from background noise in the radio signals [15]. AZEL stands for Azimuth and Elevation.

- distance to the shower axis;

- angle to the geomagnetic field;

- azimuthal and zenith angle of the shower.

Since electrons and positrons are responsible for the radiosynchrotron signal generation, the radio signal should only depend on the total number of electrons and positrons (the 'electron number') in the shower. However - due to propagation effects in the atmosphere and the way of measurement - the recorded radio signal also depends on the azimuthal angle, the zenith angle, and the fact that only one polarisation is measured. In particular, due to the generation mechanism, the dependence of the radio yield on the angle of the shower axis with respect to the geomagnetic field plays a special role.

It is not easy to disentangle the various correlations for these dependencies. When, for example, the dependence of the radio pulse height on the geomagnetic angle is to be extracted, the dependence of the radio signal on the shower size and the lateral distance of the shower must be considered and corrected for. In the same way, the dependence of the radio signal on the electron or muon number must take into account that geomagnetic angle and core distance effects have to be corrected for. In the following it is tried to present various functional dependencies of two parameters each where the correlation with other parameters is taken into account.

The analysis of LOPES data, as presented in the following, is based on primaries with energies above $5 \cdot 10^{16} \mathrm{eV}$ which are observed by the smaller KASCADE array and LOPES in coincidence. The efficiency for radio detection by 
LOPES - as derived from measurements in coincidence with the KASCADE-Grande array [10] - depends on the primary energy. It is appoximately $60 \%$ for primaries with energies exceeding $2 \cdot 10^{17} \mathrm{eV}$. There is a number of reasons that this efficiency cannot be $100 \%$. Firstly, there is a detection threshold for radio showers which, among others, depends on the ambient radio background. Secondly, the present LOPES detection system is sensitive to only one polarization direction, and thirdly, if the shower is incident along the direction of the geomagnetic field, there will be very little synchrotron emission.

The following results are based on a sample of events that have been collected from January to September 2004 [9]. Out of 630000 events coincident with KASCADE 556626 'good' data from KASCADE have been found. Since the efficiency for radio detection is strongly dependent on the primary energy, only high energy showers have been selected. For the subsample of energetic showers 412 'good' KASCADE events were selected, out of which 228 had a clear radio peak, corresponding to an efficiency for this sample of $55 \%$. For these LOPES 10 events the radio pulse height normalised to the muon number (i.e. essentially to the shower size or equivalent primary energy determined from the muon data) and distance to the shower axis shows a clear correlation with the geomagnetic angle (figure 8).

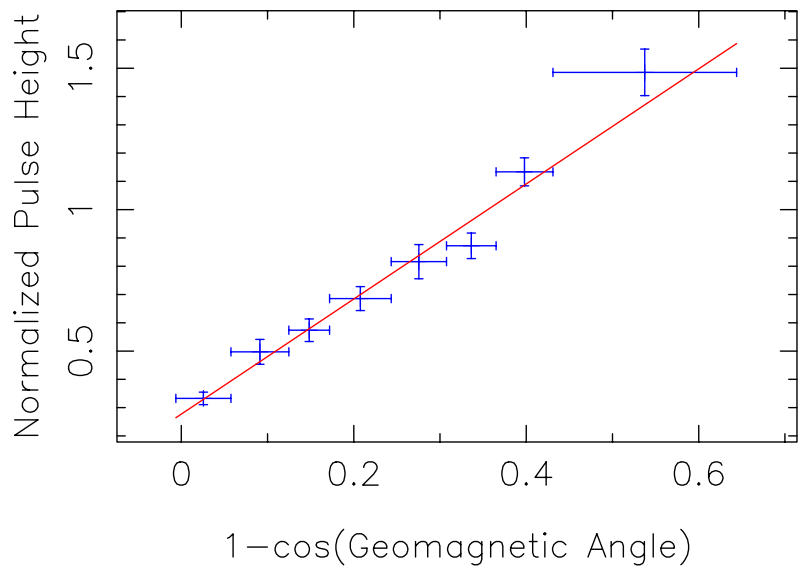

FIG. 8: Radio pulse height normalised to the muon number and distance to the shower axis as a function of the geomagnetic angle in terms of $1-\cos \alpha[9]$.

Primaries incident parallel to the geomagnetic field $(\cos \alpha=1)$ can produce very little synchrotron radiation because the transverse momenta of shower particles are usually very small.

If the muon data are corrected for the geomagnetic angle effect a clear correlation of the radio pulse height with the shower size in terms of the muon number is observed (figure 9).

A correlation of the radio signal with the electron number in the shower is also observed, but it is less pronounced compared to the muon case (figure 10).

This may be related to the following: Even though the radio signal originates from all phases of the longitudinal development, and considering the fact that the signals are atten-

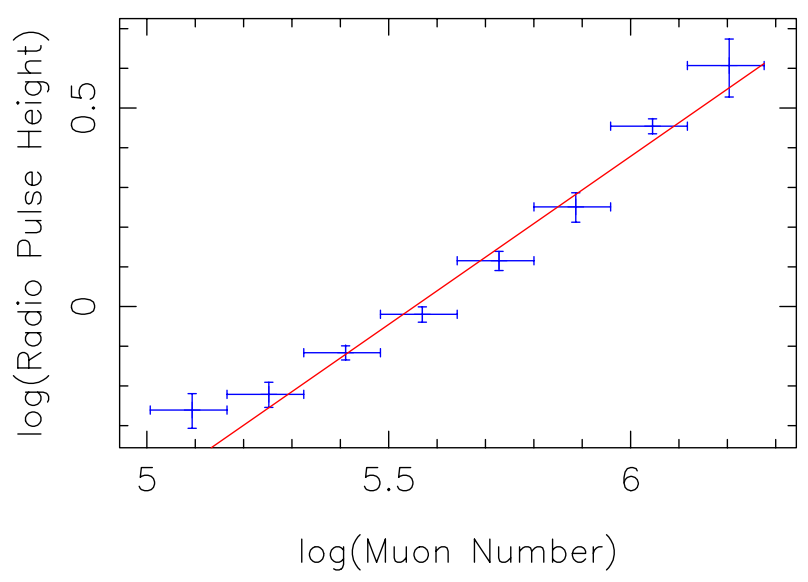

FIG. 9: Variation of the radio pulse height with the muon size of the shower after the signal amplitude has been corrected for the geomagnetic angle and the distance to the shower core [9]. The experimental point in the first bin is biased by the efficiency for a poor signal-tonoise ratio at low shower sizes.

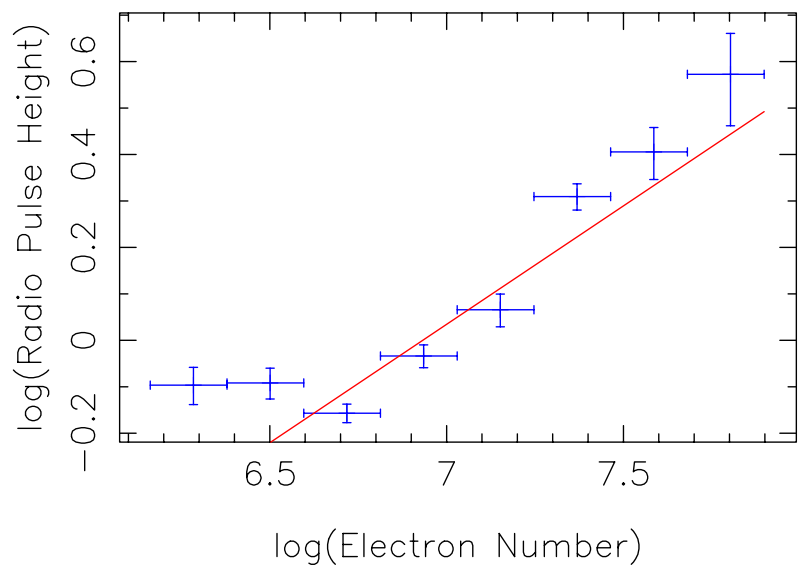

FIG. 10: Variation of the radio pulse height with the electron size of the shower after the signal amplitude has been corrected for the geomagnetic angle and the distance to the shower core [9].

uated in different ways depending on where they are generated, the radio signal mainly originates from the large number of particles in the shower maximum while the observed electron number is given predominantly by the local production in the tail of the atmospheric shower. On the other hand, muons present in the shower maximum can penetrate down to ground level because of their small energy loss. These features are not unexpected for the observed energy range accessible to the KASCADE and KASCADE-Grande experiments, also with respect to the fact that these experiments are operating essentially at sea level where most of the showers are far beyond their shower maximum. For these reasons it is expected that the muon number is a better tracer of the shower energy. Another uncertainty that will certainly take an influence on the correlations is the unknown primary particle mass, because the shower size in terms of muons and electrons does depend on the atomic mass of the primary particle initiating the shower. Also, the missing zenith angle correction might 
have an impact on the correlation of the radio signal with the electron and muon number.

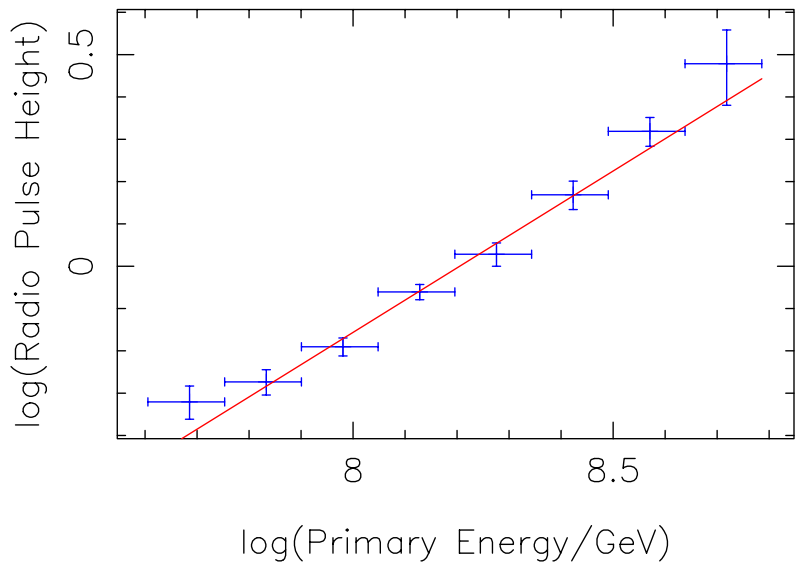

FIG. 11: Variation of the radio pulse height with the primary particle energy of the shower as derived from the electron and muon size after the signal amplitude has been corrected for the geomagnetic angle and distance to the shower core [9].

Finally, figure 11 shows the dependence of the radio pulse height on the primary particle energy, which has been derived from the combined electron and muon number of the shower.

The analysis of the available statistics of radio signals for KASCADE and KASCADE-Grande triggered and selected events leads to the following results:

- angular resolution of the shower axis of better than $1^{\circ}$;

- width of the radio cone of a few degrees;

- duration of the radio flash of $\leq 45 \mathrm{~ns}$.

For completeness the dependence of the radio pulse height on the distance from the shower axis is presented in figure 12 .

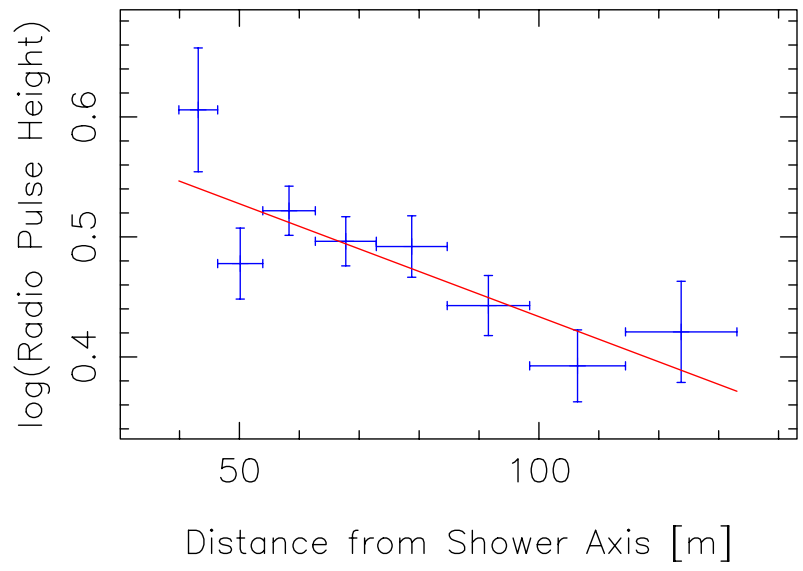

FIG. 12: Variation of the normalised radio pulse height with distance from the shower axis after scaling for geomagnetic angle and shower size effects [9].

The radio pulse is coherent (the thickness of the shower disk is smaller than the wavelength of the emitted radio wave), the pulse height scales like a power law with a spectral index of 1 with the shower energy, and it is correlated with the geomagnetic angle, which is a clear indication that the production mechanism is geosynchrotron radiation.

The discussion of the zenith-angle dependence of the radio signal is somewhat intricate. For the energy range in question the total number of electrons depends very little on the zenith angle, because the shower maximum is relatively high in the atmosphere. Therefore the radio signal should also not depend significantly on the zenith angle. On the other hand, the recorded shower size at sea level does depend on the zenith angle, since the absorption of the electrons is much stronger than the attenuation of the radio signal. This leads to a smaller recorded shower size, producing less noise for the radio detection, making the radio signal look cleaner, because of the improved signal-to-noise ratio. Also, for a fixed trigger threshold, inclined showers are on average of higher energy, and since they are 'old', the 'footprint' of the radio signal is smeared over a larger area, thereby easing radio detection in a widely spaced array [19]. In addition, for larger zenith angles the angle with respect to the geomagnetic field becomes more favorable. If all these interdependencies are considered, it is believed that the radio pulse height should show no clear dependence neither on the zenith angle nor on the azimuthal angle if the radio signal is scaled with the muon number and the effect of the geomagnetic field is taken into account. This behaviour is also confirmed by the data [9].

\section{SUMMARY AND CONCLUSIONS}

LOPES has verified the phenomenon of geosynchrotron emission of extensive air showers. With digital filtering and cross-correlated beam forming the radio pulses can be measured even in a radio loud environment. The radio pulse height depends - as expected - on the geomagnetic angle. After geomagnetic angle correction the radio pulse height is strongly correlated with the muon number, and also with the electron number, even though the correlation with the muon number is somewhat more pronounced. This means that the radio pulse height scales with the shower energy. In principle, the radio measurements also allow to determine the shower core position, the angle of emission and the shower width. Still there is room for improvement towards a detailed understanding of the interplay of the different shower parameters.

One must, however, remember that the LOPES results have so far been obtained in an offline analysis based on selected air shower data triggered by a classical air shower sampling array. A stand-alone radio detection array, like LOFAR [20], still needs to verify the results obtained so far.

The better duty cycle of radio detection, compared to air fluorescence techniques, and the integration over the full longitudinal development, compared to ground-level sampling is reason enough to forecast that radio detection is an attractive and cost-effective alternative for air shower measurements at high primary energies. 


\section{ACKNOWLEDGEMENTS}

The authors of the LOPES Collaboration would like to thank the engineering and technical staff of the involved institutes. The corresponding author (C.G.) expresses his thanks to the organisers of the XXVI Brasilian National Meeting on
Particles and Fields (BNMPF), in particular to Fernando Marroquim Leao de Almeida Jr., for the relaxed atmosphere at São Lourenço and the hospitality extended to him. The corresponding author also acknowledges the careful reading of the manuscript by Tilo Stroh.
[1] C. Grupen, Astroparticle Physics, Springer, Heidelberg, 2005

[2] T. Antoni et al., KASCADE Collaboration, Nucl. Instr. Meth. A 513, 429 (2003)

[3] M. Takeda et al., Astropart. Phys. 19, 447 (2003)

[4] J.N. Matthews et al., Proc. Int. Cosmic Ray Conf. 2001, and http://hires.physics.utah.edu/

[5] Auger Collaboration, http://www.auger.org/

[6] G. A. Askaryan, Soviet Physics JETP 14, 441 (1962)

[7] P.W. Gorham, SLAC Summer Institute on Particle Physics (SSI04), Aug. 2-13 (2004)

[8] F.D. Kahn, I. Lerche, Royal Society of London, Proceedings Series A 289, 206 (1966)

[9] A. Horneffer, Ph.D. thesis, Measuring Radio Emission from Cosmic Ray Air Showers with a Digital Radio Telescope, Max-Planck-Institut für Radioastronomie Bonn, and Universität Bonn, Germany (2006)

[10] W.D. Apel et al., LOPES Collaboration, submitted to Astropart. Phys. 2006

[11] A.F. Badea et al., Forschungszentrum Karlsruhe, Wis- senschaftliche Berichte, FZKA 7229, April 2006

[12] J.V. Jelley et al., Nature 205, 237 (1965)

[13] H.R. Allan, Progr. in Element. Part. and Cosmic Ray Physics, Vol. 10, 171 (1971)

[14] S.A. Colgate, J. Geophys. Res. 72, 4869 (1967)

[15] H. Falcke et al. - LOPES Collaboration, Nature 435, 313 (2005)

[16] H. Falcke, P.W. Gorham, Astropart. Phys. 19, 477 (2003)

[17] T. Huege, H. Falcke, Astron. and Astroph. 412, 19 (2003)

[18] T. Huege, H. Falcke, Astron. and Astroph. 430, 779 (2005)

[19] T. Huege, H. Falcke, Astropart. Phys. 24, 116 (2005)

[20] LOFAR Collaboration, http://www.lofar.org/

[21] G. Navarra et al., KASCADE-Grande Collaboration, Nucl. Instr. Meth. A 513, 429 (2003)

[22] J. Petrovic et al., LOPES Collaboration, submitted to Astronomy and Astrophysics, June 2006

[23] H. Falcke, Radio Detection of Cosmic Rays with LOPES, ASTRON, Dwingeloo, The Netherlands, Radboud University, Nijmegen and the LOPES Collaboration, Talk 2004 